\title{
Calibration of the Volatility in Option Pricing Using the Total Variation Regularization
}

\author{
Yu-Hua Zeng, ${ }^{1,2}$ Shou-Lei Wang, ${ }^{1,2}$ and Yu-Fei Yang ${ }^{3}$ \\ ${ }^{1}$ College of Mathematics and Econometrics, Hunan University, Changsha 410082, China \\ ${ }^{2}$ Department of Mathematics, Hunan First Normal University, Changsha 410205, China \\ ${ }^{3}$ Department of Information and Computing Science, Changsha University, Changsha 410003, China
}

Correspondence should be addressed to Yu-Hua Zeng; cszyh@hnu.edu.cn

Received 6 November 2013; Revised 27 January 2014; Accepted 24 February 2014; Published 25 March 2014

Academic Editor: Roberto Renò

Copyright (C) 2014 Yu-Hua Zeng et al. This is an open access article distributed under the Creative Commons Attribution License, which permits unrestricted use, distribution, and reproduction in any medium, provided the original work is properly cited.

\begin{abstract}
In market transactions, volatility, which is a very important risk measurement in financial economics, has significantly intimate connection with the future risk of the underlying assets. Identifying the implied volatility is a typical PDE inverse problem. In this paper, based on the total variation regularization strategy, a bivariate total variation regularization model is proposed to estimate the implied volatility. We not only prove the existence of the solution, but also provide the necessary condition of the optimal control problem-Euler-Lagrange equation. The stability and convergence analyses for the proposed approach are also given. Finally, numerical experiments have been carried out to show the effectiveness of the method.
\end{abstract}

\section{Introduction}

Volatility is a very important risk measurement in financial economics. The estimation of it is critical for option pricing and management of the derivative positions. In order to estimate the volatility effectively, two main classes of parametric approaches have been developed: discrete-time models and continuous-time models.

There are numerous literatures on the discrete-time models and here we provide only a partial overview related to our studies. The ARCH model developed by Engle [1] is the first model that provided a systematic framework for volatility modeling. Based on the ARCH model, Bollerslev [2] proposed the GARCH model and Nelson [3] and Glosten et al. [4] argued that the GARCH model provides more flexibility. There are many popular extensions including EGARCH [3], GJR-GARCH [4], QGARCH [5], TGARCH [6], and GARCH-M [7]. Moreover, a multifactors volatility structure has been studied in Engel and Lee [8], Christoffersen et al. [9], Li and Zhang [10], and Adrian and Rosenbery [11]. On the other hand, models for asset pricing under risk-neutral measure have been dominated traditionally by continuoustime processes. Heston [12] proposed an option pricing model with stochastic volatility. Duan [13] and Heston and Nandi [14] developed an option pricing model based on the GARCH process. However, those models fail to address the smile and the smirk quantitatively. Existing literatures have attempted to cope with this by combining stochastic volatility specifications with jump process or by using nonnormal innovations in GARCH models; see, for example, Bates [15, 16], Pan [17], Duan et al. [18, 19], Eraker [20], Broadie et al. [21], Christoffersen et al. [22-24], and so forth.

However, those models generally suffer from a curse of dimension that severely constrains their practice and the coming of high frequency financial data makes it worse. Nowadays, the availability of intraday data has facilitated the use of the so-called Realized Volatility (RV) which was introduced in the literature by Taylor and $\mathrm{Xu}$ [25] and Anderson and Bollerslev [26] and which is grounded in the framework of continuous time finance with the notion of quadratic variation of a martingale. The literature on RV models has grown remarkably over the last decade; see, for example, Andersen et al. [27], Andersen et al. [28, 29], Barndorff-Nielsen and Shephard [30, 31], Bandi et al. [32], and references therein. The RV model has the clear advantage of providing a precise nonparametric measure of daily 
volatility which leads to simplicity in model estimation and superior forecasting performance. Corsi et al. [33] followed a similar approach by jointly modeling returns and the two-scale realized volatility [34]. Christoffersen et al. [35] developed a new class of affine discrete-time models that allow for closed-form option valuation formulas using the conditional moment generation function and modeled daily returns as well as realized volatility.

There is also a common practice to infer the volatility from quoted option prices based on the Black-Scholes theoretical framework [36], called implied volatility; see, for example, Dupier [37], Lagnado and Osher [38], Chiarella et al. [39], Jiang and Tao [40], Crépey [41], Isakov [42], Egger and Engl [43], Ngnepieba [44], Deng et al. [45], and so forth. The volatility value implied by an observed market option price (implied volatility) indicates a consensual view about the volatility level determined by the market. This paper is devoted to studying the regularization method of identifying the implied volatility.

The stochastic process of the asset price $S_{t}$ is modeled to satisfy the Geometric Brownian motion:

$$
d S_{t}=\mu S_{t} d t+\sigma S_{t} d \omega(t),
$$

where $\mu$ is the expected rate of return, $\sigma$ is the volatility, and $\omega(t)$ is the standard Brownian process; here $E\left[\omega(t)^{2}\right]=t$.

An option is classified either as a call option or a put option. A call (put) option is a contract which gives the buyer (the owner) the right, but not the obligation, to buy (or sell) an underlying asset or instrument at a specified strike price on or before a specified date.

Suppose $V\left(S_{t}, t\right)$ is the price of a European option, the differential of which is given by

$$
d V=\left(\frac{\partial V}{\partial S} \mu S+\frac{\partial V}{\partial t}+\frac{1}{2} \sigma^{2} S^{2} \frac{\partial^{2} V}{\partial S^{2}}\right) d t+\frac{\partial V}{\partial S} \sigma S d w(t) .
$$

Consider a portfolio that involves short selling of one unit of a European call option and long holding of $\Delta_{t}$ units of the underlying asset. The portfolio value $\Pi\left(S_{t}, t\right)$ at time $t$ is given by

$$
\Pi=-V+\Delta_{t} S .
$$

By virtue of the no-arbitrage principle, we have

$$
\begin{gathered}
\frac{\partial V}{\partial t}+r S \frac{\partial V}{\partial S}+\frac{1}{2} \sigma^{2} S^{2} \frac{\partial^{2} V}{\partial S^{2}}=r V \\
V_{T}=\left(S_{T}-K\right)^{+}=\max \left(S_{T}-K, 0\right), \text { call option } \\
V_{T}=\left(K-S_{T}\right)^{+}=\max \left(K-S_{T}, 0\right), \text { put option, }
\end{gathered}
$$

where $r$ is the riskless interest rate, $T$ is the maturity, and $K$ is the strike price. The above parabolic partial differential equation is the famous Black-Scholes equation. With the boundary condition $V(0, t)=0$, that is, the option is worthless if the stock is valued at nothing, the analytical solution of the European call option is given by

$$
V(S, t)=S N\left(d_{1}\right)-K e^{-r(T-t)} N\left(d_{2}\right),
$$

where

$$
\begin{aligned}
N(x) & =\frac{1}{\sqrt{2 \pi}} \int_{-\infty}^{x} e^{-\omega^{2} / 2} d \omega \\
d_{1} & =\frac{\ln (S / K)+\left(r+\left(\sigma^{2} / 2\right)\right)(T-t)}{\sigma \sqrt{(T-t)}} \\
d_{2} & =d_{1}-\sigma \sqrt{(T-t)}
\end{aligned}
$$

The option prices obtained from the Black-Scholes pricing model are functions of five parameters: $S, K, r, T$, and $\sigma$. Except for the volatility parameter, the other four parameters $T, K, q$, and $r$ are observable quantities. There is evidence that the volatility is time varying $[46,47]$ in actual markets. For any fixed maturity, implied volatility varies with the strike price in a parabolic shape that is often called the volatility smile. The pattern of implied volatilities across maturities is known as the volatility term structure. One possibility to explain the volatility smiles in the Black-Scholes model is to use a deterministic function of underlying asset price $S_{t}$ and time $t$; that is, $\sigma=\sigma(S, t)$.

A natural question then arises: how can we get the implied volatility of the future underlying asset by option quotes? This is the typical IPOP (inverse problem of option pricing).

The PDE inverse problem of option pricing was first considered by Dupire in [37] where he obtained a formula of the local volatility with all possible strike prices and maturities. However, the formula was instable and could not be used in practice. The inverse problem which consists in using the results of actual measurements to infer the values of the parameters is usually ill-posed. The fact that the solution fails to depend continuously upon the given data is the source of many difficulties inherently in solving the inverse problem. Ill-posed problems require the use of regularization techniques for any practical application. The most widely known and applicable regularization methods is Tikhonov regularization [48], where regular items play a critical role of stability. Over the past decades, the inverse problem of determining the implied volatility has already obtained widespread development; see, for example, [38-45, 49] and references therein. However, the traditional Tikhonov regularization strategy may oversmooth the solution, so that the regularized solution cannot effectively approximate the exact solution of the original problem, when the exact solution is nonsmooth or even has some singularities. This shortcoming will blur the edge of the restored image in image processing. To overcome the shortcoming, Rudin et al. [50] proposed the total variation regularization strategy (TV- $L^{2}$ model):

$$
\min _{u \in \Omega} \frac{\lambda}{2}\|u-f\|_{L^{2}(\Omega)}^{2}+|\nabla u|_{L^{1}(\Omega)} .
$$

The total variation regularization might be able to characterize the properties (the jump, overnight, weekend effect, etc.) of the volatility better. So whether the total variation regularization strategy could be applied to identify the implied volatility is a question worth pondering.

This paper is organized as follows. Section 2 introduces the total variation regularization item in the inverse problem 
of option pricing and puts forward a new model with terminal observations. In Section 3, we give mathematical analysis of the existence of the solution and the necessary condition of the optimal control problem. The stability and convergence of the proposed regularized approach are analyzed in Section 4. Section 5 presents a selection of numerical experiments. Section 6 concludes the paper.

\section{Total Variation Regularization Model}

In [38] Lagnado and Osher determined this inverse problem by using Tikhonov regularization strategy that is attempting to minimize

$$
\widetilde{G}(\sigma)=\frac{1}{2} \sum_{i=1}^{N} \sum_{j=1}^{M_{i}}\left(V\left(S_{0}, 0, K_{i j}, T_{i}, \sigma\right)-V_{i j}\right)^{2}+\|\nabla \sigma\|_{2}^{2}
$$

where $\nabla$ denotes the gradient operator. This regularization strategy is just for one fixed value of underlying asset $S_{0}$, at one fixed point at time $t=0$. There is no guarantee that the value of $\sigma$ calculated by this approach will be correct either for other underlying assets or at future times, and the estimated volatility may be negative in some cases.

Based on their work, Chiarella et al. [39] modified the objective functional as follow:

$$
\begin{aligned}
\widehat{G}(\sigma)= & \frac{1}{2} \sum_{i=1}^{N} \sum_{j=1}^{M_{i}} \int_{0}^{\infty} \int_{0}^{T_{\text {cur }}}\left[V\left(S, t, K_{i j}, T_{i}, \sigma\right)-V_{i j}\right]^{2} \\
& d S d t+\|\nabla \sigma\|_{2}^{2},
\end{aligned}
$$

where $T_{\text {cur }}$ is the current time.

Tikhonov regularization strategy may oversmooth the solution, so it may not preserve the singularities of the solution well. We adopt total variation regularization strategy proposed by Rudin et al. [50] to maintain the singularity (the jump, overnight, weekend effect, etc.) of volatility. In fact, total variation regularization strategy can preserve the edge of the restored image and has become a standard approach for the computation of discontinuous solutions of inverse problems.

Set $V(S, t, \sigma(S, t))$ (hereafter denote $V(S, t, \sigma(S, t))$ by $V(\sigma)$ for convenience sake) to be the solution of the Black-Scholes equations (4) and (5); then we regard $V(\sigma)$ as a nonlinear operator with respect to $\sigma$ :

$$
L^{2}(\Omega) \supseteq \mathscr{D} \ni \sigma \stackrel{V}{\longrightarrow} V(\sigma) \in L^{2}(\Omega) .
$$

Consider the following bivariate total variation regularization problem:

$$
\min _{\sigma \in \mathscr{D}} \widehat{J}(\sigma)=\frac{1}{2}\|V(\sigma)-v\|^{2}+\alpha J(\sigma)
$$

where $J(\sigma)$ is the seminorm

$$
J(\sigma)=\int_{\Omega}|\nabla \sigma| d S d t
$$

$\alpha$ denotes the regularization parameter, and $v$ is the vector of market observed prices at the calibration time. $\mathscr{D}: \mathscr{D}(J) \neq \emptyset$ for "operator" $V(\sigma), \Omega:\left(0, S_{\max }\right) \times\left(0, T_{\text {cur }}\right]$ and

$$
\begin{aligned}
\mathscr{D}(J) & :=\{\sigma \in \Lambda: J(\sigma) \neq \infty\}, \\
\Lambda & =\left\{\sigma \equiv \sigma(S, t) \mid 0 \leq \sigma_{\min } \leq \sigma \leq \sigma_{\max }, \sigma \in L^{2}(\Omega)\right\},
\end{aligned}
$$

where $\sigma_{\min }, \sigma_{\max }$ are given constants.

The term $|\nabla \sigma|^{-1}$ will appear in later necessary optimality condition. To avoid $|\nabla \sigma| \approx 0$ in the flat area, as is done in the image processing, the problem (13) is usually approximated by

$$
\min _{\sigma \in D} \bar{J}(\sigma)=\frac{1}{2}\|V(\sigma)-v\|^{2}+\alpha J_{\beta}(\sigma)
$$

where

$$
J_{\beta}(\sigma)=\int_{\Omega} \sqrt{|\nabla \sigma|^{2}+\beta^{2}} d S d t
$$

$\beta$ is a (typically small) positive parameter which usually can be taken as a constant, for example, $\beta=10^{-6}$.

Our total variation regularization strategy has two advantages compared with Tikhonov regularization strategy proposed by Lagnado and Osher: one is that it contains no terms involving the Dirac delta function [51]; the other is that the total variation regularization strategy may maintain the singularities of the solution better. Next we will investigate mathematical properties of the solution such as the existence, necessary condition, stability, and convergence.

\section{Existence and Necessary Optimality Condition}

The minimization problem (16) is quite different from the standard Tikhonov regularization strategy since the regularization item involves $J_{\beta}(\sigma)$.

Lemma 1. Under the constraints of the total variation regularization problem (16), if $\left\{\sigma_{n}\right\} \rightarrow \sigma^{*}$, then $\left\{V\left(\sigma_{n}\right)\right\} \rightarrow V\left(\sigma^{*}\right)$, where $V\left(\sigma_{n}\right)$ is the solution to (4) when $\sigma=\sigma_{n}$.

This lemma can easily be similarly proved like proposition A.3 in [43].

Theorem 2. The total variation minimization problem (16) at least attains a minimizer $\widetilde{\sigma} \in \mathscr{D}$.

Proof. The weak lower semicontinuity of the norm and weakly continuity of the operator $V(\sigma)$ imply the lower semicontinuity of the functionals $\|V(\sigma)-v\|^{2}$ and $J_{\beta}(\sigma)$. Moreover, the level sets of the functional $J_{\beta}(\sigma)$ are compact in $L^{2}(\Omega)$. So the total variation minimization problem (16) has a compact set of minimizers by Theorem 2 in [48]. 
We can calculate approximate solutions by solving the Euler-Lagrange equation. Generally speaking, the total variational regularization problem (16) is not strictly convex or even nonconvex. Next we deduce the necessary condition Euler-Lagrange equation which has to be satisfied by each optimal control minimum.

Set

$$
F=\frac{1}{2}[V(\sigma)-v]^{2}+\alpha|\nabla \sigma(S, t)|,
$$

and further assume that $F$ is the third-order differentiable function and $\sigma=\sigma(S, t)$ is the second-order differentiable function.

Theorem 3. Necessary optimality condition: let $\sigma$ be a solution of the total variation regularization problem (16); then $\sigma$ satisfies

$$
\frac{\partial V}{\partial \sigma}(S, t, \sigma)[V(\sigma)-v]-\alpha \nabla \cdot\left(\frac{\nabla \sigma}{\sqrt{|\nabla \sigma|^{2}+\beta^{2}}}\right)=0 .
$$

Proof. By using the variational method, the corresponding Euler-Lagrange partial differential equation is

$$
F_{\sigma}-\frac{\partial}{\partial S}\left\{F_{p}\right\}-\frac{\partial}{\partial t}\left\{F_{q}\right\}=0
$$

where

$$
p=\frac{\partial \sigma(S, t)}{\partial S}, \quad q=\frac{\partial \sigma(S, t)}{\partial t} .
$$

Combining (18) and (20), we have

$$
\begin{gathered}
F_{\sigma}=\frac{\partial V}{\partial \sigma}(S, t, \sigma)[V(\sigma)-v], \\
F_{p}=\frac{\partial \sigma / \partial S}{|\nabla \sigma|}, \quad F_{q}=\frac{\partial \sigma / \partial t}{|\nabla \sigma|} .
\end{gathered}
$$

Therefore

$$
\begin{aligned}
\frac{\partial V}{\partial \sigma}(S, t, \sigma)[V(\sigma)-v] & -\alpha\left\{\frac{\partial}{\partial S}\left\{\left(\frac{\partial \sigma / \partial S}{|\nabla \sigma|}\right)\right\}+\frac{\partial}{\partial t}\left\{\left(\frac{\partial \sigma / \partial t}{|\nabla \sigma|}\right)\right\}\right\}=0 \\
\Longrightarrow & \frac{\partial V}{\partial \sigma}(S, t, \sigma)[V(\sigma)-v] \\
& -\alpha\left(\frac{\partial}{\partial S}, \frac{\partial}{\partial t}\right) \cdot\left\{\frac{\partial \sigma / \partial S}{|\nabla \sigma|}, \frac{\partial \sigma / \partial t}{|\nabla \sigma|}\right\}=0 \\
\Longrightarrow & \frac{\partial V}{\partial \sigma}(S, t, \sigma)[V(\sigma)-v] \\
& -\alpha\left(\frac{\partial}{\partial S}, \frac{\partial}{\partial t}\right) \cdot\left\{\frac{1}{|\nabla \sigma|}\left(\frac{\partial \sigma}{\partial S}, \frac{\partial \sigma}{\partial t}\right)\right\}=0 \\
\Longrightarrow & \frac{\partial V}{\partial \sigma}(S, t, \sigma)[V(\sigma)-v]-\alpha \nabla \cdot\left(\frac{\nabla \sigma}{|\nabla \sigma|}\right)=0
\end{aligned}
$$

The corresponding Euler-Lagrange equation related to the total variation model with $|\nabla \sigma|$ replaced by $|\nabla \sigma|_{\beta}$ is given by

$$
\frac{\partial V}{\partial \sigma}(S, t, \sigma)[V(\sigma)-v]-\alpha \nabla \cdot\left(\frac{\nabla \sigma}{\sqrt{|\nabla \sigma|^{2}+\beta^{2}}}\right)=0 .
$$

This completes the proof.

The next theorem states well posedness of the regularized problem.

Theorem 4. Under the constraints of the total variation regularization problem (16), the minimization of

$$
\bar{J}_{\beta}^{\delta}(\sigma)=\frac{1}{2}\left\|V(\sigma)-v^{\delta}\right\|^{2}+\alpha J_{\beta}(\sigma)
$$

is stable with respect to perturbations in the data; that is, $\alpha>$ 0 , if $\left\{v^{k}\right\} \rightarrow v^{\delta}$ in $L^{2}(\Omega)$ and $\sigma_{k}$ denotes the solution to the problem (25) with $v^{\delta}$ replaced by $v^{k}$, then

$$
\left\{\sigma_{k}\right\} \longrightarrow \sigma, \quad\left\{J_{\beta}\left(\sigma_{k}\right)\right\} \rightarrow J_{\beta}(\sigma) .
$$

Proof. $\left\{v^{k}\right\} \rightarrow v^{\delta}$ in $L^{2}(\Omega)$ implies that $\left\{\sigma_{k}, V_{k}\left(\sigma_{k}\right)\right\}$ satisfies

$$
\begin{aligned}
& \frac{1}{2}\left\|V_{k}\left(\sigma_{k}\right)-v^{k}\right\|^{2}+\alpha J_{\beta}\left(\sigma_{k}\right) \\
& \quad \leq \frac{1}{2}\left\|V(\sigma)-v^{k}\right\|^{2}+\alpha J_{\beta}(\sigma)
\end{aligned}
$$

for every $\sigma \in \mathscr{D}$. Thus $\left\{\sigma_{k}\right\}$ is bounded in $\mathscr{D}$ and therefore has a weakly convergent subsequence $\left\{\sigma_{m}\right\} \rightarrow \widehat{\sigma}$. Similarly, there exists a subsequence $\left\{V_{m}\right\}$ corresponding to $\left\{\sigma_{m}\right\}$ such that $\left\{V_{m}\right\} \rightarrow \widehat{V}$, where $\widehat{V}$ is the solution to (4) when $\sigma=\widehat{\sigma}$. By the weak lower semicontinuity of $J_{\beta}\left(\sigma_{m}\right)$ and $\|\cdot\|$, we have

$$
\begin{aligned}
J_{\beta}(\widehat{\sigma}) & \leq \limsup J_{\beta}\left(\sigma_{m}\right), \\
\frac{1}{2}\left\|\widehat{V}-v^{\delta}\right\|^{2} & \leq \limsup \frac{1}{2}\left\|V_{m}-v^{m}\right\|^{2},
\end{aligned}
$$

and therefore by (27),

$$
\begin{aligned}
\frac{1}{2} \| \widehat{V} & -v^{\delta} \|+\alpha J_{\beta}(\widehat{\sigma}) \\
& \leq \liminf \left\{\frac{1}{2}\left\|V_{m}-v^{m}\right\|^{2}+\alpha J_{\beta}\left(\sigma_{m}\right)\right\} \\
& \leq \limsup \left\{\frac{1}{2}\left\|V_{m}-v^{m}\right\|^{2}+\alpha J_{\beta}\left(\sigma_{m}\right)\right\} \\
& \leq \lim _{m \rightarrow \infty}\left\{\frac{1}{2}\left\|V-v^{m}\right\|^{2}+\alpha J_{\beta}(\sigma)\right\} \\
& =\frac{1}{2}\left\|V-v^{\delta}\right\|^{2}+\alpha J_{\beta}(\sigma)
\end{aligned}
$$

for all $\sigma \in \mathscr{D}$. This implies that $\widehat{\sigma}$ is a minimizer of the total variation regularization problem (25) and that

$$
\begin{gathered}
\lim _{m \rightarrow \infty}\left\{\frac{1}{2}\left\|V_{m}-v^{m}\right\|^{2}+\alpha J_{\beta}\left(\sigma_{m}\right)\right\} \\
=\frac{1}{2}\left\|\widehat{V}-v^{\delta}\right\|^{2}+\alpha J_{\beta}(\widehat{\sigma}) .
\end{gathered}
$$


If $\left\{\sigma_{m}, V_{m}\right\}$ does not converge strongly to $\{\widehat{\sigma}, \widehat{V}\}$, then

$$
C:=\lim \sup \left\{\frac{1}{2}\left\|V_{m}-v^{\delta}\right\|^{2}\right\}>\frac{1}{2}\left\|\widehat{V}-v^{\delta}\right\|^{2},
$$

and there exists a subsequence $\left\{\sigma_{n}, V_{n}\right\}$ of $\left\{\sigma_{m}, V_{m}\right\}$ satisfying

$$
\begin{gathered}
\sigma_{n} \rightarrow \widehat{\sigma}, \quad V_{n} \rightarrow \widehat{V}, \quad J_{\beta}(\widehat{\sigma}) \leq \lim _{n \rightarrow \infty} J_{\beta}\left(\sigma_{n}\right), \\
\frac{1}{2}\left\|V_{n}-v^{\delta}\right\|^{2} \longrightarrow C .
\end{gathered}
$$

This combined with (30) implies

$$
\alpha \lim _{n \rightarrow \infty} J_{\beta}\left(\sigma_{n}\right)=\alpha J_{\beta}(\widehat{\sigma})+\frac{1}{2}\left\|\widehat{V}-v^{\delta}\right\|^{2}-C<\alpha J_{\beta}(\widehat{\sigma}),
$$

which is a contradiction to (32), so we have

$$
\left\{\sigma_{n}\right\} \longrightarrow \widehat{\sigma}, \quad J_{\beta}\left(\sigma_{n}\right) \longrightarrow J_{\beta}(\widehat{\sigma}) .
$$

This completes the proof.

In the next theorem, we show that under the same conditions on $\alpha(\delta)$ as in the linear case solutions of (25) converge to a minimum-norm-solution, that is, a least squares solution.

Theorem 5. Under the constraints of the total variation regularization problem (16), there exists at least one minimizing solution of (25). Assume that the sequence $\left\{\delta_{k}\right\}$ converges monotonically to 0 and $v^{k}:=v^{\delta_{k}}$ satisfies $\left\|v-v^{k}\right\| \leq \delta_{k}$; here $v$ denotes the solution of the Black-Scholes model with respect to the minimum solution.

Moreover, assume that $\alpha(\delta)$ satisfies

$$
\alpha(\delta) \longrightarrow 0, \quad \frac{\delta^{2}}{\alpha(\delta)} \longrightarrow 0 \quad \text { as } \delta \longrightarrow 0,
$$

and $\alpha(\cdot)$ is monotonically increasing. Then every sequence $\left\{\sigma_{\alpha_{k}}^{\delta_{k}}\right\}$, where $\delta_{k} \rightarrow 0, \alpha_{k}:=\alpha\left(\delta_{k}\right)$,

$$
\sigma_{\alpha_{k}}^{\delta_{k}} \in \operatorname{argmin}\left\{\left\|V(\sigma)-v^{k}\right\|^{2}+\alpha J(\sigma): \sigma \in \mathscr{D}\right\},
$$

has a convergent subsequence. The limit of every convergent subsequence is a minimum solution. If, in addition, the minimum solution $\sigma^{+}$is unique, then

$$
\lim _{\delta \rightarrow 0} \sigma_{\alpha(\delta)}^{\delta}=\sigma^{+} .
$$

Proof. Let $\alpha_{k}$ and $\left\{\sigma_{\alpha_{k}}^{\delta_{k}}\right\}$ be as above, and let $\sigma^{+}$be a minimum solution. Then by the definition of $\left\{\sigma_{\alpha_{k}}^{\delta_{k}}\right\}$, we have

$$
\begin{aligned}
& \left\|V\left(\sigma_{\alpha_{k}}^{\delta_{k}}\right)-v^{k}\right\|^{2}+\alpha_{k} J\left(\sigma_{\alpha_{k}}^{\delta_{k}}\right) \\
& \leq\left\|V\left(\sigma^{+}\right)-v^{k}\right\|^{2}+\alpha_{k} J\left(\sigma^{+}\right) \\
& =\delta_{k}^{2}+\alpha_{k} J\left(\sigma^{+}\right),
\end{aligned}
$$

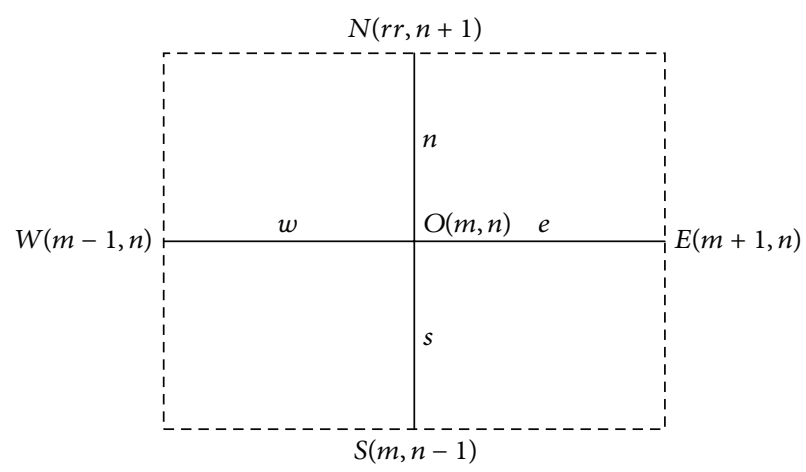

FIGURE 1: Gridding.

which shows that

$$
\begin{gathered}
\lim _{k \rightarrow \infty} V\left(\sigma_{\alpha_{k}}^{\delta_{k}}\right)=v, \\
\limsup _{k \rightarrow \infty} J\left(\sigma_{\alpha_{k}}^{\delta_{k}}\right) \leq J\left(\sigma^{+}\right) .
\end{gathered}
$$

This combined with (15) implies that $\left\{\sigma_{\alpha_{k}}^{\delta_{k}}\right\}$ is bounded. Hence, there exist an element $\dot{\sigma} \in \mathscr{D}$ and a subsequence again denoted by $\left\{\sigma_{\alpha_{k}}^{\delta_{k}}\right\}$ such that

$$
\sigma_{\alpha_{k}}^{\delta_{k}} \rightarrow \dot{\sigma} \quad \text { as } k \longrightarrow \infty .
$$

Using the assumption that $V(\sigma)$ is continuous with respect to $\sigma$ and that the norm convergence on $L^{2}(\Omega)$ is stronger, it follows from (40) that $V(\dot{\sigma})=v$.

From the lower semicontinuity of $J(\sigma)$, it follows that

$$
J(\dot{\sigma}) \leq \liminf _{k \rightarrow \infty} J\left(\sigma_{\alpha_{k}}^{\delta_{k}}\right) \leq \limsup _{k \rightarrow \infty} J\left(\sigma_{\alpha_{k}}^{\delta_{k}}\right) \leq J\left(\sigma^{+}\right) \leq J(\bar{\sigma}),
$$

for all $\bar{\sigma} \in \mathscr{D}$ satisfying $V(\bar{\sigma})=v$. Taking $\bar{\sigma}=\dot{\sigma}$ shows that $J(\dot{\sigma})=J\left(\sigma^{+}\right)$. That means $\dot{\sigma}$ is a minimizing solution of the total variation regularization problem.

Using this and (42), it follows that $J\left(\sigma_{\alpha_{k}}^{\delta_{k}}\right) \rightarrow J\left(\sigma^{+}\right)$.

If the minimizing solution of (25) is unique denoted by $\sigma^{+}$, it follows that every sequence $\left\{\sigma_{\alpha_{k}}^{\delta_{k}}\right\}$ has a subsequence, and the limit of any subsequence of $\left\{\sigma_{\alpha_{k}}^{\delta_{k}}\right\}$ has to be equal to $\sigma^{+}$. This completes the proof.

\section{Discretization and Algorithm}

Next we will discretize the term $\nabla \cdot\left(\nabla \sigma / \sqrt{|\nabla \sigma|^{2}+\beta^{2}}\right)$. Let $\{\Delta S, \Delta t\}$ denote the grid size and construct an approximation for $\sigma(S, t)$ at a set of points $(m \Delta S, n \Delta t)$ on $\Omega$.

As in Figure 1, at a given target pixel $O(m, n)$ (we denote $O(m \Delta S, m \Delta t)$ by $O(m, n)$ for convenience sake), let $E, N, W$, and $S$ denote its four adjoint pixels, and let $e, n, w$, and $s$ be the corresponding four midway points (not directly available from the gridding). 
Let $v=\left(v^{1}, v^{2}\right)=\nabla \sigma /|\nabla \sigma|_{\beta}$; then

$$
\nabla \cdot v=\frac{\partial v^{1}}{\partial S}+\frac{\partial v^{2}}{\partial t} \approx \frac{v_{e}^{1}-v_{w}^{1}}{\Delta S}+\frac{v_{n}^{2}-v_{s}^{2}}{\Delta t}
$$

Next, we give further approximations at the midway points:

$$
\begin{gathered}
v_{e}^{1}=\frac{1}{\left|\nabla \sigma_{e}\right|_{\beta}}\left[\frac{\partial \sigma}{\partial S}\right]_{e} \approx \frac{1}{\left|\nabla \sigma_{e}\right|_{\beta}} \frac{\sigma_{E}-\sigma_{O}}{\Delta S} ; \\
\left|\nabla \sigma_{e}\right|_{\beta} \approx \sqrt{\left(\frac{\sigma_{E}-\sigma_{O}}{\Delta S}\right)^{2}+\left(\frac{\sigma_{N E}+\sigma_{N}-\sigma_{S}-\sigma_{S E}}{4 \Delta t}\right)^{2}+\beta^{2} .}
\end{gathered}
$$

Namely, we approximate $[\partial \sigma / \partial S]_{e}$ by the central difference scheme and $[\partial \sigma / \partial t]_{e}$ by the average of $\left(\sigma_{N E}-\sigma_{S E}\right) / 2 \Delta t$ and $\left(\sigma_{N}-\sigma_{S}\right) / 2 \Delta t$. Similar discussion applies to the other three directions $N, W$, and $S$ :

$$
\begin{aligned}
& v_{w}^{1}=\frac{1}{\left|\nabla \sigma_{w}\right|_{\beta}}\left[\frac{\partial \sigma}{\partial S}\right]_{w} \approx \frac{1}{\left|\nabla \sigma_{w}\right|_{\beta}} \frac{\sigma_{W}-\sigma_{O}}{\Delta S} ; \\
&\left|\nabla \sigma_{w}\right|_{\beta} \approx \sqrt{\left(\frac{\sigma_{W}-\sigma_{O}}{\Delta S}\right)^{2}+\left(\frac{\sigma_{N W}+\sigma_{N}-\sigma_{S}-\sigma_{S W}}{4 \Delta t}\right)^{2}+\beta^{2}} ; \\
& v_{n}^{2}=\frac{1}{\left|\nabla \sigma_{n}\right|_{\beta}}\left[\frac{\partial \sigma}{\partial t}\right]_{n} \approx \frac{1}{\left|\nabla \sigma_{n}\right|_{\beta}} \frac{\sigma_{N}-\sigma_{O}}{\Delta t} ; \\
&\left|\nabla \sigma_{n}\right|_{\beta} \approx \sqrt{\left(\frac{\sigma_{N}-\sigma_{O}}{\Delta t}\right)^{2}+\left(\frac{\sigma_{N E}+\sigma_{E}-\sigma_{W}-\sigma_{W N}}{4 \Delta S}\right)^{2}+\beta^{2}} ; \\
& v_{s}^{2}=\frac{1}{\left|\nabla \sigma_{s}\right|_{\beta}}\left[\frac{\partial \sigma}{\partial t}\right]_{s} \approx \frac{1}{\left|\nabla \sigma_{s}\right|_{\beta}} \frac{\sigma_{S}-\sigma_{O}}{\Delta t} ; \\
&\left|\nabla \sigma_{s}\right|_{\beta} \approx \sqrt{\left(\frac{\sigma_{S}-\sigma_{O}}{\Delta t}\right)^{2}+\left(\frac{\sigma_{E S}+\sigma_{E}-\sigma_{W}-\sigma_{W S}}{4 \Delta S}\right)^{2}+\beta^{2}},
\end{aligned}
$$

and then we have

$$
\begin{aligned}
-\nabla \cdot\left(\frac{\nabla \sigma}{|\nabla \sigma|_{\beta}}\right)= & -\nabla \cdot v=\left(\frac{v_{w}^{1}-v_{e}^{1}}{\Delta S}\right)+\left(\frac{v_{s}^{2}-v_{n}^{2}}{\Delta t}\right) \\
= & \sum_{p \in W, E} \frac{1}{\left|\nabla \sigma_{p}\right|_{\beta}}\left[\frac{\sigma(O)-\sigma(p)}{(\Delta S)^{2}}\right] \\
& +\sum_{p \in N, S} \frac{1}{\left|\nabla \sigma_{p}\right|_{\beta}}\left[\frac{\sigma(O)-\sigma(p)}{(\Delta t)^{2}}\right] .
\end{aligned}
$$

At a pixel $O(m, n),(19)$ is discretized to

$$
\begin{aligned}
0= & \sum_{i=1}^{I} \sum_{j=1}^{J} \frac{\partial V}{\partial \sigma}\left(m \Delta S, n \Delta t, K_{i}, T_{j}, \sigma(m \Delta S, n \Delta t)\right) \\
& \times\left[V\left(m \Delta S, n \Delta t, K_{i}, T_{j}, \sigma(m \Delta S, n \Delta t)\right)-v_{i j}\right] \\
& +\alpha \sum_{p \in W, E} \frac{1}{\left|\nabla \sigma_{p}\right|_{\beta}}\left[\frac{\sigma(m \Delta S, n \Delta t)-\sigma(p)}{(\Delta S)^{2}}\right] \\
& +\alpha \sum_{p \in N, S} \frac{1}{\left|\nabla \sigma_{p}\right|_{\beta}}\left[\frac{\sigma(m \Delta S, n \Delta t)-\sigma(p)}{(\Delta t)^{2}}\right] .
\end{aligned}
$$

To obtain the local optimal solution, we have to handle the problem of calculating the partial derivative $\partial V / \partial \sigma$ in the Euler-Lagrange equation. By the Black-Scholes formula, the option price $V$ and partial derivative $\partial V / \partial \sigma$ can be approximated, respectively, as follows:

$$
\begin{aligned}
C(S, t) & =S N\left(d_{1}\right)-K e^{-r(T-t)} N\left(d_{2}\right), \\
\frac{\partial C}{\partial \sigma} & =S N^{\prime}\left(d_{1}\right) \frac{\partial d_{1}}{\partial \sigma}-K e^{-r(T-t)} N^{\prime}\left(d_{2}\right) \frac{\partial d_{2}}{\partial \sigma} \\
& =\frac{S \sqrt{T-t} e^{-d_{1}^{2} / 2}}{\sqrt{2 \pi}} .
\end{aligned}
$$

Let

$$
\begin{aligned}
A:= & \{W, E\}, \quad B:=\{N, S\}, \\
A_{p 1}= & \alpha \sum_{p \in A} \frac{1}{\left|\nabla_{p}\right|_{\beta}(\Delta S)^{2}}, \quad B_{p 2}=\alpha \sum_{p \in B} \frac{1}{\left|\nabla \sigma_{p}\right|_{\beta}(\Delta t)^{2}}, \\
C= & \sum_{i=1}^{I} \sum_{j=1}^{J} \frac{\partial V}{\partial \sigma}\left(m \Delta S, n \Delta t, K_{i}, T_{j}, \sigma(m \Delta S, n \Delta t)\right) \\
& \times\left[V\left(m \Delta S, n \Delta t, K_{i}, T_{j}, \sigma(m \Delta S, n \Delta t)\right)-v_{i j}\right],
\end{aligned}
$$

and then we have

$$
\sigma(m \Delta S, n \Delta t)=\frac{A_{p 1} \sigma(p 1)+B_{p 2} \sigma(p 2)-C}{A_{p 1}+B_{p 2}} .
$$

We adopt the Gauss-Jacobi iteration scheme. At each step $k$, we update $\sigma^{k-1}$ to $\sigma^{k}$ by

$$
\sigma^{k}(m \Delta S, n \Delta t)=\frac{A_{p 1}^{k-1} \sigma^{k-1}(p 1)+B_{p 2}^{k-1} \sigma^{k-1}(p 2)-C^{k-1}}{A_{p 1}^{k-1}+B_{p 2}^{k-1}} .
$$

An important issue in practice is the choice of the regularization parameter $\alpha$, which determines the balance between accuracy and regularity in the method. In general, the smaller the $\alpha$, the preciser the solution. When $\alpha \rightarrow 0$, the optimal control functional can reach the exact solution but is unstable. So regularization parameter $\alpha$ should not be too big 
so that the process of seeking $\sigma_{\alpha}$ is stable. There are two main approaches to set $\alpha$. One is a priori methods, in which the choice of $\alpha$ only depends on $\delta$, the level on noise on the data, such as the size of bid-ask spread; the other is a posteriori methods, in which $\alpha$ may depend on the data in a less specific way. In financial literatures the most commonly used method for choosing $\alpha$ is the a posteriori methods based on the socalled discrepancy principle (such as Morozov discrepancy principle [52]), which consists in choosing the greatest level of $\alpha$ for which the data fidelity item does not exceed the level of noise $\delta$ on the observations:

$$
\alpha:=\sup \left\{\alpha>0,\left\|V(\sigma)-v^{\delta}\right\|<d \delta\right\} .
$$

Algorithm 6. Total variation for solving the implied volatility.

(1) Choose a function $\sigma_{0}(S, t)$. This will be the initial approximation to the true volatility.

(2) Determine $\sigma_{0}(m \Delta S, n \Delta t)$.

(3) Compute $V\left(m \Delta S, n \Delta t, K_{i}, T_{j}, \sigma^{k}(m \Delta S, n \Delta t)\right)$ and $(\partial V / \partial \sigma)\left(m \Delta S, n \Delta t, K_{i}, T_{j}, \sigma^{k}(m \Delta S, n \Delta t)\right)$ by using the Black-Scholes formula:

$$
\begin{aligned}
C(S, t) & =S N\left(d_{1}\right)-K e^{-r(T-t)} N\left(d_{2}\right), \\
\frac{\partial C}{\partial \sigma} & =S N^{\prime}\left(d_{1}\right) \frac{\partial d_{1}}{\partial \sigma}-K e^{-r(T-t)} N^{\prime}\left(d_{2}\right) \frac{\partial d_{2}}{\partial \sigma} \\
& =\frac{S \sqrt{T-t} e^{-d_{1}^{2} / 2}}{\sqrt{2 \pi}} .
\end{aligned}
$$

(4) Compute $A_{p 1}^{k}, B_{p 2}^{k}, C^{k}, \sigma^{k}(p 1)$, and $\sigma^{k}(p 2)$.

(5) Adopt the Gauss-Jacobi iteration scheme:

$$
\sigma^{k+1}(m \Delta S, n \Delta t)=\frac{A_{p 1}^{k} \sigma^{k}(p 1)+B_{p 2}^{k} \sigma^{k}(p 2)-C^{k}}{A_{p 1}^{k}+B_{p 2}^{k}} .
$$

(6) If $\left\|\sigma^{k+1}-\sigma^{k}\right\|_{\infty}<$ tol, the iteration is stopped; otherwise $k=k+1$ and go to step 3 .

\section{Numerical Experiments}

In this section, we present numerical experiments to illustrate the theory and algorithm presented in above sections. First we assume that the true volatility function, $\sigma_{e x}(S, t)$, is defined as

$$
\sigma_{e x}(S, t)=\left\{\begin{array}{c}
0.2+0.01 e^{-0.01 S}+\frac{\cos (3 t)}{100} \\
0.19+0.01 e^{-0.01 S}+\frac{\cos (3 t)}{100}
\end{array}\right.
$$

In numerical experiments, the interest rate $r=0.05$, $S_{\max }=100$, we consider only one time to option maturity $T=1$. We take $\Delta S=1, \Delta t=0.01$ and $K_{1}=40, K_{2}=$ $41, \ldots K_{21}=60$. Figure 2 displays the true volatility function.

We solve the volatility by using Algorithm 6, and Figure 3 shows the error between $\sigma_{e x}(S, t)$ and the estimated $\sigma_{\mathrm{TV}}^{50}(S, t)$,

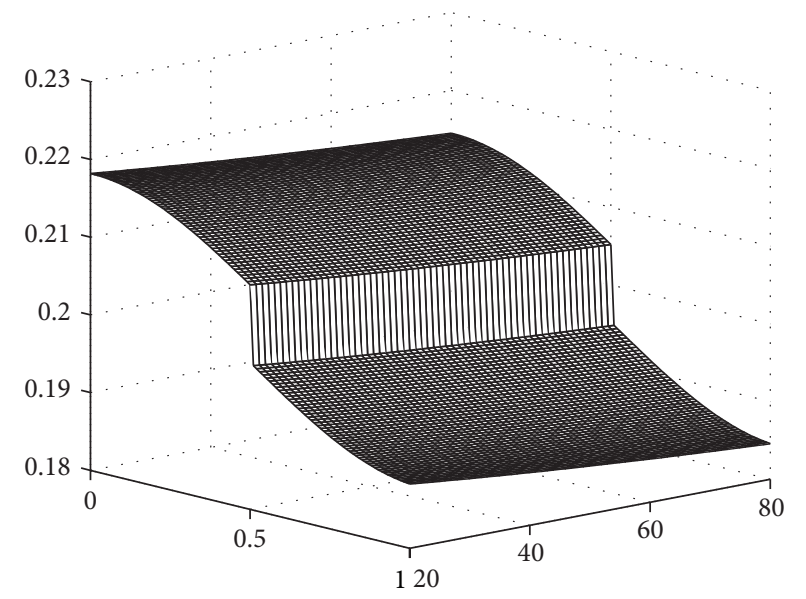

Figure 2: Volatility function $\sigma_{e x}(S, t)$.

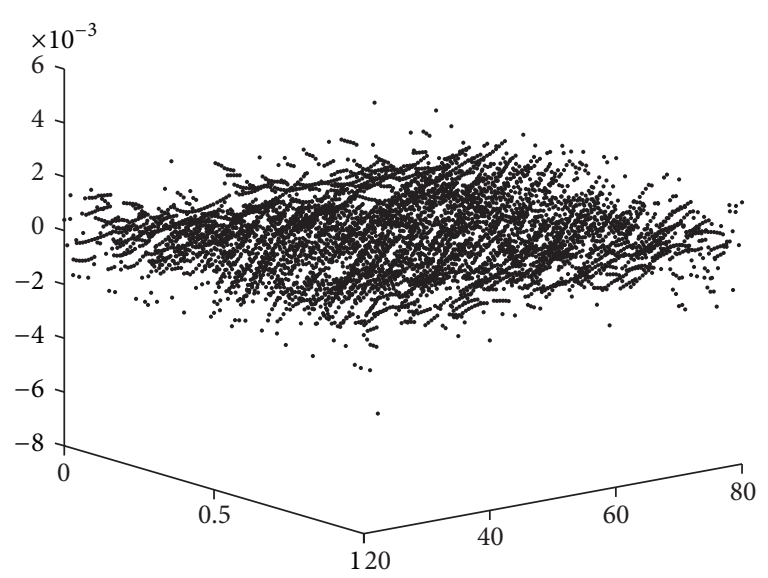

FIGURE 3: The error between $\sigma_{e x}(S, t)$ and $\sigma_{\mathrm{TV}}^{50}(S, t)$.

where $\sigma_{\mathrm{TV}}^{50}(S, t)$ denotes the 50 iterations of total variation algorithm. Almost all errors fell in the region $[-0.002,0.002]$ and $\left\|\sigma_{\mathrm{TV}}^{50}(S, t)-\sigma_{e x}(S, t)\right\|_{\infty}=0.0069$.

If we fix $S=S^{*}$, for example, $S^{*}=40$, Figure 4 shows the comparison between $\sigma_{e x}(40, t)$ (continuous line) and $\sigma_{\mathrm{TV}}^{50}(40, t)$. Figure 5 shows the comparison between the $\sigma_{e x}(40, t)$ and $\sigma_{\text {TIK }}^{50}(40, t)$ (by using the classical Tikhonov regularization strategy) and $\left\|\sigma_{\mathrm{TIK}}^{50}(S, t)-\sigma_{e x}(S, t)\right\|_{\infty}=0.0161$.

According to Figures 4 and 5, the estimation of implied volatility using total variation regularization has two advantages compared with the classical Tikhonov regularization: one is that the total variation regularization maintains the singularities of the solution better (when $T=0.5$ ) and the Tikhonov regularization oversmooths the discontinuity point; the other is that the error $\left(\left\|\sigma(S, t)-\sigma_{e x}(S, t)\right\|_{\infty}\right)$ obtained by total variation regularization is smaller.

\section{Conclusion}

A lot of research works have been made to determine the implied volatility by regularization strategies. Based on the 


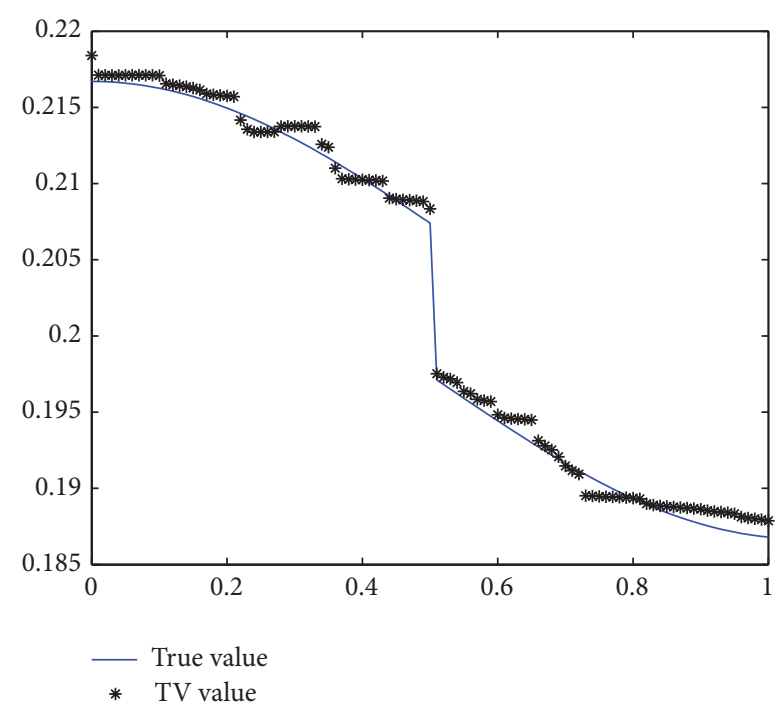

FIGURE 4: $\sigma_{e x}(40, t)$ and $\sigma_{\mathrm{TV}}^{50}(40, t)$.

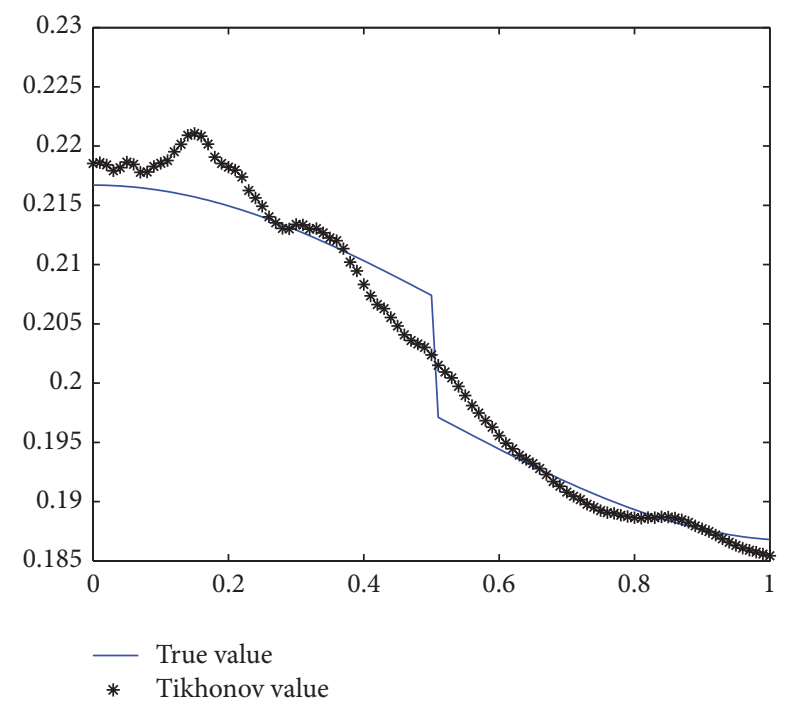

Figure 5: $\sigma_{e x}(40, t)$ and $\sigma_{\mathrm{TIK}}^{50}(40, t)$.

advantages and great success of the total variation regularization strategy in image processing, we propose the total variation regularization strategy to estimate the implied volatility under the framework of the Black-Scholes model. We identify the implied volatility by solving an optimal control problem and investigate a rigorous mathematical analysis. Not only the existence is discussed, but also the stability and convergence for this regularized approach are given. We also deduce the Euler-Lagrange equation. Furthermore, the results of numerical experiments are presented.

\section{Conflict of Interests}

The authors declare that there is no conflict of interests regarding the publication of this paper.

\section{Acknowledgments}

This work was supported by the NNSF of China (nos. 60872129, 11271117) and Science and Technology Project of Changsha City of China (no. K1207023-31).

\section{References}

[1] R. F. Engle, "Autoregressive conditional heteroscedasticity with estimates of the variance of United Kingdom inflation," Econometrica, vol. 50, no. 4, pp. 987-1007, 1982.

[2] T. Bollerslev, "Generalized autoregressive conditional heteroskedasticity," Journal of Econometrics, vol. 31, no. 3, pp. 307327, 1986.

[3] D. B. Nelson, "Conditional heteroskedasticity in asset returns: a new approach," Econometrica, vol. 59, no. 2, pp. 347-370, 1991.

[4] L. R. Glosten, R. Jagannathan, and D. Runkle, "On the relation between the expected value and the volatility of the nominal excess return on stocks," The Journal of Finance, vol. 48, pp. 1779-1801, 1993.

[5] E. Sentana, "Quadratic ARCH models," Review of Economic Studies, vol. 62, pp. 639-661, 1995.

[6] J.-M. Zakoian, “Threshold heteroskedastic models," Journal of Economic Dynamics and Control, vol. 18, no. 5, pp. 931-955, 1994.

[7] R. F. Engle, D. M. Lilien, and R. P. Robins, "Estimating timevarying risk premia in the term structure: the ARCH-M model," Econometrica, vol. 55, pp. 391-407, 1987.

[8] R. F. Engle and G. G. J. Lee, "A permanent and transitory component model of stock return volatility," in Cointegration, Causality, and Forecasting: A Festschrift in Honor of Clive W. J. Granger, R. Engle and H. White, Eds., pp. 475-497, Oxford University Press, 1999.

[9] P. Christoffersen, R. Elkamhi, B. Feunou, and K. Jacobs, “Option valuation with conditional heteroskedasticity and nonnormality," Review of Financial Studies, vol. 23, no. 5, pp. 2139-2183, 2010.

[10] G. Li and C. Zhang, "On the number of state variables in options pricing," Management Science, vol. 56, no. 11, pp. 2058-2075, 2010.

[11] T. Adrian and J. Rosenberg, "Stock returns and volatility: pricing the short-run and long-run components of market risk," Journal of Finance, vol. 63, no. 6, pp. 2997-3030, 2008.

[12] S. L. Heston, "A closed-form solution for options with stochastic volatility with applications to bond and currency options," Review of Financial Studies, vol. 6, pp. 327-343, 1993.

[13] J.-C. Duan, “The GARCH option pricing model," Mathematical Finance, vol. 5, no. 1, pp. 13-32, 1995.

[14] S. L. Heston and S. Nandi, "A closed-form GARCH option valuation model," Review of Financial Studies, vol. 13, no. 3, pp. 585-625, 2000.

[15] D. S. Bates, "Post-87 crash fears in the S\&P 500 futures option market," Journal of Econometrics, vol. 94, no. 1-2, pp. 181-238, 2000.

[16] D. S. Bates, "Maximum likelihood estimation of latent affine processes," Review of Financial Studies, vol. 19, no. 3, pp. 909965, 2006.

[17] J. Pan, “The jump-risk premia implicit in options: evidence from an integrated time-series study," Journal of Financial Economics, vol. 63, no. 1, pp. 3-50, 2002.

[18] J. C. Duan, "Conditionally fat-tailed distributions and the volatility smile in options," Working Paper, Department of 
Finance, The Hong Kong University of Science and Technology, 1999.

[19] J.-C. Duan, P. Ritchken, and Z. Sun, "Approximating GARCHjump models, jump-diffusion processes, and option pricing," Mathematical Finance, vol. 16, no. 1, pp. 21-52, 2006.

[20] B. Eraker, "Do stock prices and volatility jump? Reconciling evidence from spot and option prices," Journal of Finance, vol. 59, no. 3, pp. 1367-1403, 2004.

[21] M. Broadie, M. Chernov, and M. Johannes, "Model specification and risk premia: evidence from futures options," Journal of Finance, vol. 62, no. 3, pp. 1453-1490, 2007.

[22] P. Christoffersen, C. Dorion, K. Jacobs, and Y. Wang, "Volatility components, affine restrictions, and nonnormal innovations," Journal of Business \& Economic Statistics, vol. 28, no. 4, pp. 483502, 2010.

[23] P. Christoffersen, S. Heston, and K. Jacobs, "Option valuation with conditional skewness," Journal of Econometrics, vol. 131, no. 1-2, pp. 253-284, 2006.

[24] P. Christoffersen, K. Jacobs, C. Ornthanalai, and Y. Wang, "Option valuation with long-run and short-run volatility components," Journal of Financial Economics, vol. 90, no. 3, pp. 272297, 2008.

[25] S. J. Taylor and X. Xinzhong, "The incremental volatility information in one million foreign exchange quotations," Journal of Empirical Finance, vol. 4, no. 4, pp. 317-340, 1997.

[26] T. G. Andersen and T. Bollerslev, "Answering the skeptics: yes, standard volatility models do provide accurate forecasts," International Economic Review, vol. 39, no. 4, pp. 885-905, 1998.

[27] T. G. Andersen, T. Bollerslev, F. X. Diebold, and H. Ebens, "The distribution of realized stock return volatility," Journal of Financial Economics, vol. 61, no. 1, pp. 43-76, 2001.

[28] T. G. Andersen, T. Bollerslev, F. X. Diebold, and P. Labys, "The distribution of realized exchange rate volatility," Journal of the American Statistical Association, vol. 96, no. 453, pp. 42-55, 2001.

[29] T. G. Andersen, T. Bollerslev, F. X. Diebold, and P. Labys, "Modeling and forecasting realized volatility," Econometrica, vol. 71, no. 2, pp. 579-625, 2003.

[30] O. E. Barndorff-Nielsen and N. Shephard, "Econometric analysis of realized volatility and its use in estimating stochastic volatility models," Journal of the Royal Statistical Society. Series B. Statistical Methodology, vol. 64, no. 2, pp. 253-280, 2002.

[31] O. E. Barndorff-Nielsen and N. Shephard, "Econometric analysis of realized covariation: high frequency based covariance, regression, and correlation in financial economics," Econometrica, vol. 72, no. 3, pp. 885-925, 2004.

[32] F. M. Bandi, J. R. Russell, and C. Yang, "Realized volatility forecasting in the presence of time-varying noise," Journal of Business \& Economic Statistics, vol. 31, no. 3, pp. 331-345, 2013.

[33] F. Corsi, N. Fusari, and D. La Vecchia, "Realizing smiles: options pricing with realized volatility," Journal of Financial Economics, vol. 107, pp. 284-304, 2013.

[34] L. Zhang, P. A. Mykland, and Y. Aït-Sahalia, "A tale of two time scales: determining integrated volatility with noisy highfrequency data," Journal of the American Statistical Association, vol. 100, no. 472, pp. 1394-1411, 2005.

[35] P. Christoffersen, B. Feunou, K. Jacobs, and N. Meddahi, "The economic value of realized volatility: Using high-frequency returns for option valuation," Working Paper, 2012.

[36] F. Black and M. Scholes, "The pricing of options and corporate liabilities," The Journal of Political Economy, vol. 81, pp. 637-654, 1973.
[37] B. Dupire, “Pricing with a smile," Risk, vol. 7, pp. 18-20, 1994.

[38] R. Lagnado and S. Osher, "A technique for calibrating derivative security pricing models: numerical solution of an inverse problem," Journal of Computational Finance, vol. 1, pp. 13-25, 1997.

[39] C. Chiarella, M. Craddock, and N. El-Hassan, “The calibration of stock option pricing models using inverse problem methodology," QFRQ Research Papers, University of Technology, Sydney, Sydney, Australia, 2000.

[40] L. Jiang and Y. Tao, "Identifying the volatility of underlying assets from option prices," Inverse Problems, vol. 17, no. 1, pp. 137-155, 2001.

[41] S. Crépey, "Calibration of the local volatility in a trinomial tree using Tikhonov regularization," Inverse Problems, vol. 19, no. 1, pp. 91-127, 2003.

[42] V. Isakov, "The inverse problem of option pricing," in Recent Development in Theories \& Numerics: International Conference on Inverse Problems, pp. 47-55, World Scientific, Singapore, 2003.

[43] H. Egger and H. W. Engl, "Tikhonov regularization applied to the inverse problem of option pricing: convergence analysis and rates," Inverse Problems, vol. 21, no. 3, pp. 1027-1045, 2005.

[44] P. Ngnepieba, "The adjoint method formulation for an inverse problem in the generalized Black-Scholes model," Journal of Systemics Cybernetics and Informatics, vol. 4, pp. 69-77, 2006.

[45] Z.-C. Deng, J.-N. Yu, and L. Yang, "An inverse problem of determining the implied volatility in option pricing," Journal of Mathematical Analysis and Applications, vol. 340, no. 1, pp. 1631, 2008.

[46] J. R. Franks and E. S. Schwartz, "The stochastic behavior of market variance implied in the price of index options," Economics Journal, vol. 101, pp. 1460-1475, 1991.

[47] R. Heynen, "An empirical investigation of observed smile patterns," Review Futures Markets, vol. 13, pp. 317-353, 1994.

[48] A. N. Tikhonov, A. S. Leonov, and A. G. Yagola, Nonlinear IllPosed Problems, vol. 14, Chapman \& Hall, London, UK, 1998.

[49] H. E. Leland, "Option pricing and replication with transaction costs," The Journal of Finance, vol. 40, pp. 1283-1301, 1985.

[50] L. I. Rudin, S. Osher, and E. Fatemi, "Nonlinear total variation based noise removal algorithms," Physica D: Nonlinear Phenomena, vol. 60, no. 1-4, pp. 259-268, 1992.

[51] G. E. Andrews, R. Askey, and R. Roy, Special Functions, vol. 71, Cambridge University Press, Cambridge, UK, 1999.

[52] V. A. Morozov, "On the solution of functional equations by the method of regularization," Soviet Mathematics. Doklady, vol. 7, pp. 414-417, 1966. 


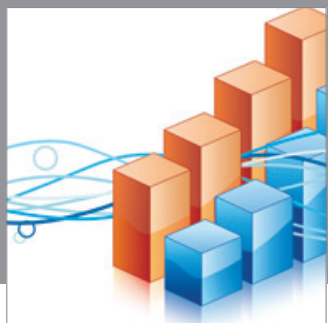

Advances in

Operations Research

mansans

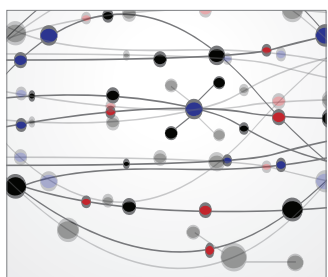

The Scientific World Journal
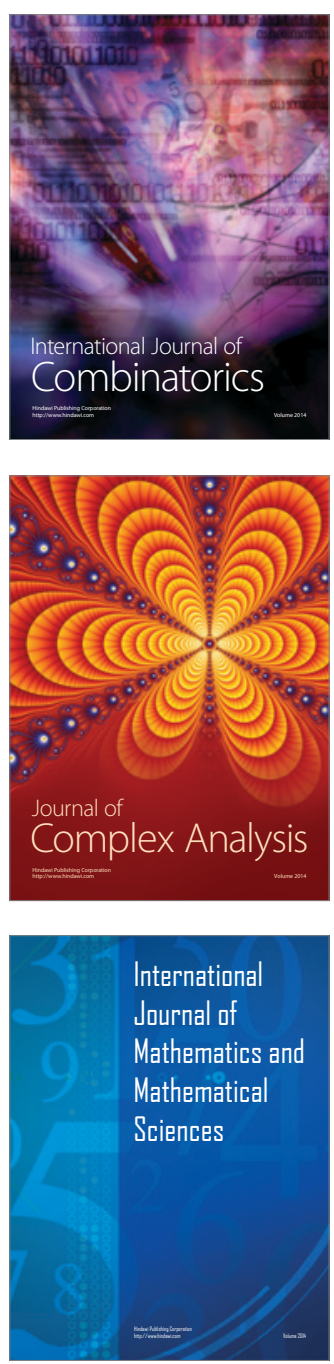
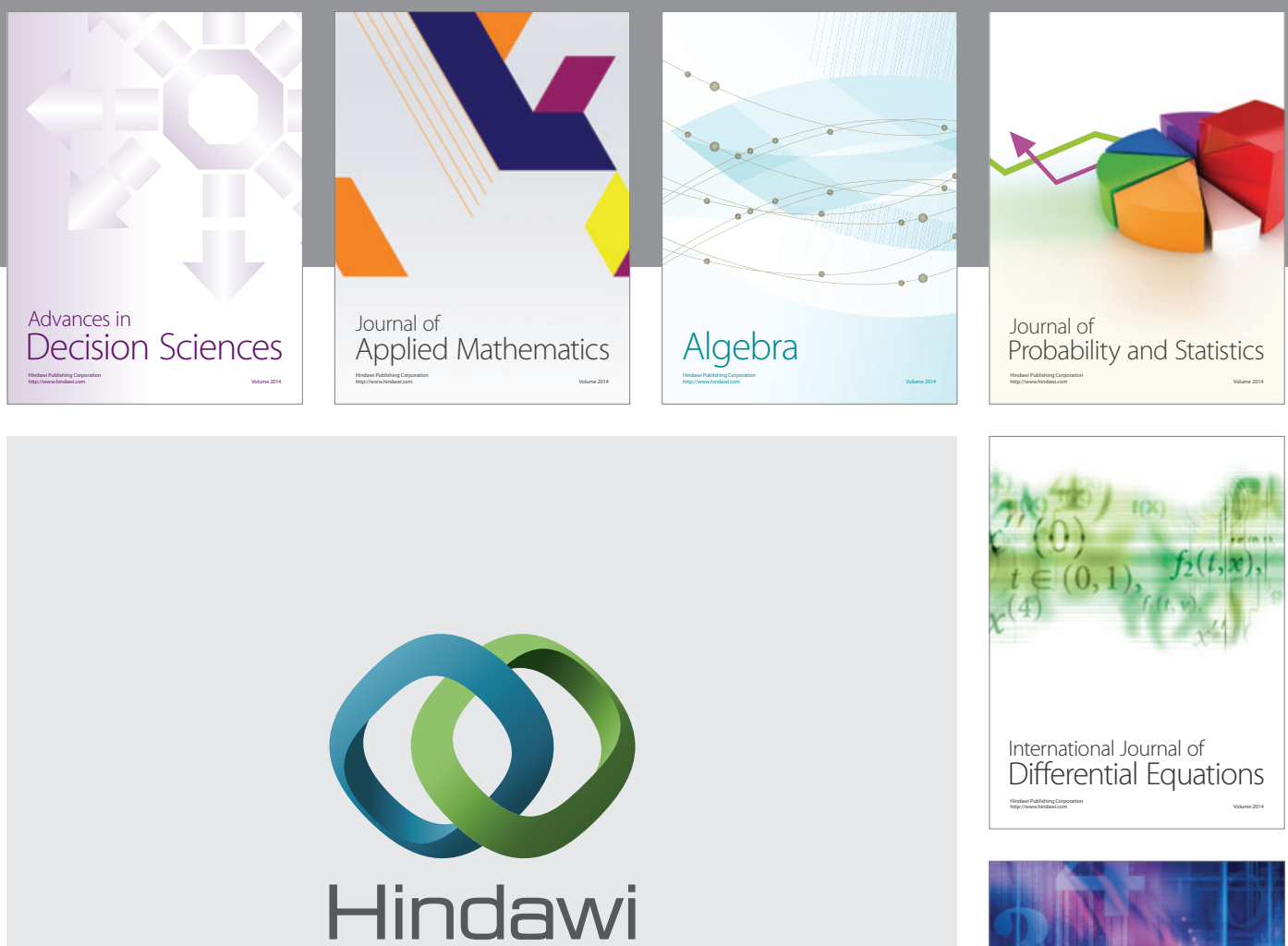

Submit your manuscripts at http://www.hindawi.com
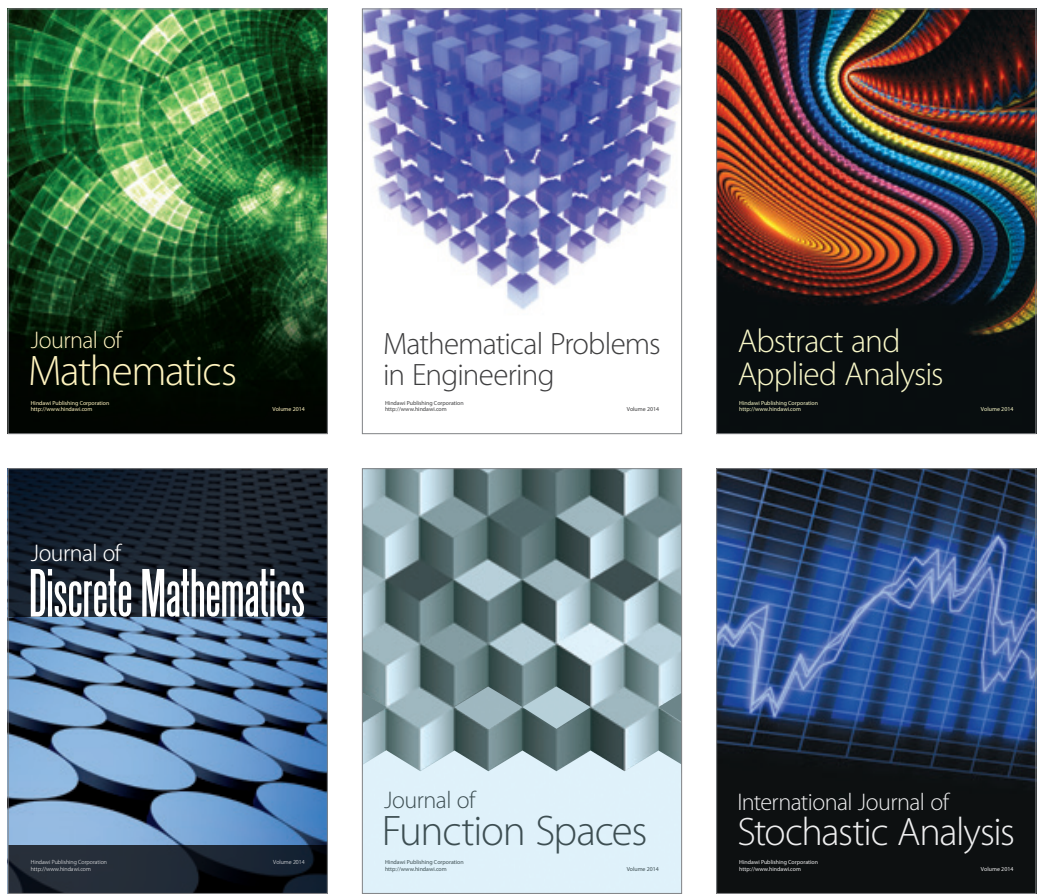

Journal of

Function Spaces

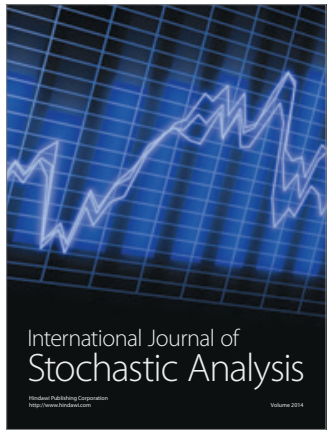

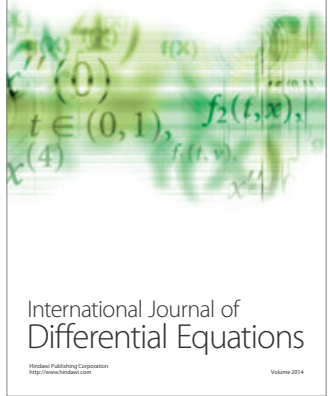
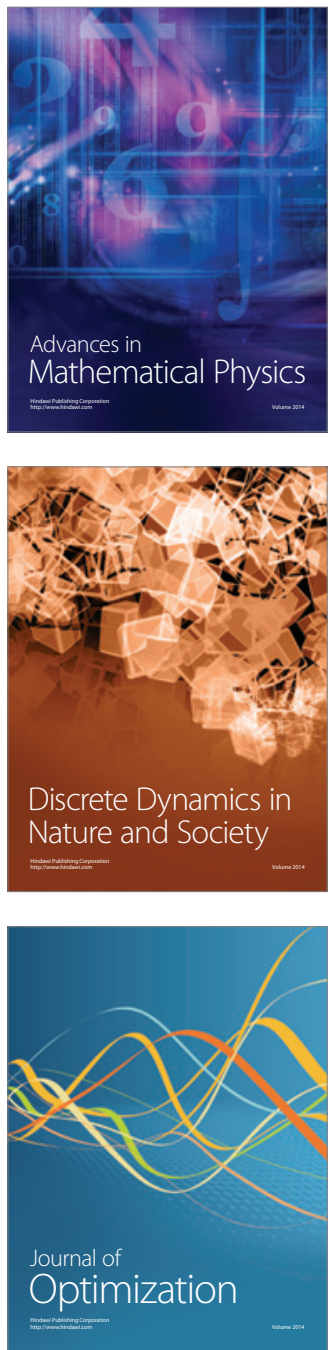